\title{
Respiratory bronchiolitis-associated interstitial lung disease with fibrosis is a lesion distinct from fibrotic nonspecific interstitial pneumonia: a proposal
}

\author{
Samuel A Yousem \\ Department of Pathology, University of Pittsburgh Medical Center, Pittsburgh, PA, USA
}

\begin{abstract}
Nine cases of clinical and radiographic chronic interstitial lung disease are presented that have features of respiratory bronchiolitis-associated interstitial lung disease, but were associated with a respiratory bronchiolitis having extensive paucicellular lamellar eosinophilic collagenous thickening of alveolar septa in a patchy, particularly subpleural distribution. Patients were middle-aged with shortness of breath, mixed obstructive and restrictive lung disease with markedly reduced diffusing capacity and radiographs demonstrating centrilobular micronodules, occasional ground glass opacities and emphysema. All were alive at follow-up. The morphology of this process raises the differential diagnosis with the fibrotic form of nonspecific interstitial pneumonia and highlights the role of cigarette smoking as a potential cause of fibrotic lung disease.
\end{abstract}

Modern Pathology (2006) 19, 1474-1479. doi:10.1038/modpathol.3800671; published online 1 September 2006

Keywords: respiratory bronchiolitis; nonspecific interstitial pneumonia; desquamative interstitial pneumonia; cigarette smoking; tobacco use

Respiratory bronchiolitis is a common incidental finding in the lungs of cigarette smokers and is manifested by an intraluminal and intra-airspace accumulation of finely pigmented macrophages associated with peribronchiolar inflammation and fibrosis. ${ }^{1-6}$ When associated with clinical symptoms of shortness of breath and cough, pulmonary function abnormalities of mixed restrictive and obstructive lung disease and high resolution CT scanning demonstrating centrilobular micronodules, ground glass opacities and peribronchiolar thickening, a diagnosis of respiratory bronchiolitisassociated interstitial lung disease can be considered. ${ }^{7-14}$ While the above small airway changes and airspace macrophages have been areas of primary pathologic interest, Fraig et al and others have also pointed out that very mild degrees of centrilobular alveolar septal and focal subpleural fibrosis can be present in nearly $50 \%$ of respiratory bronchiolitis cases. $^{6,9,15,16}$ This collagenous thickening of the

Correspondence: Dr SA Yousem, MD, Department of Pathology, University of Pittsburgh Medical Center, Presbyterian Campus, Room A610, 200 Lothrop Street, Pittsburgh, PA 15213-2582, USA. E-mail: yousemsa@upmc.edu

Received 12 May 2006; revised 5 July 2006; accepted 6 July 2006; published online 1 September 2006 alveolar septa can be dramatic and is the basis for this report of nine cases referred for consultation with the diagnosis of fibrotic phase of nonspecific interstitial pneumonia, but instead are interpreted as a form of respiratory bronchiolitis-associated interstitial lung disease with pronounced panlobular and subpleural alveolar septal fibrosis occurring in the presence of emphysematous change.

\section{Materials and methods}

The consultation files of the author were searched for all cases referred to the author in consultation as possible 'nonspecific interstitial pneumonia' from 2000 to 2006. Of these 32 cases, nine cases were reclassified as respiratory bronchiolitis-associated interstitial lung disease with fibrosis and formed the basis for this report. Hematoxylin and eosin slides were available on all cases (range 4-17 slides: average six slides per case). All nine cases were diagnosed by open lung biopsy, and trichrome stains were available in three cases. Amyloid stains were negative in four cases.

Radiographic findings and pulmonary function tests were provided by the referring pathologist and/ or pulmonologist, as was information on current patient status. 


\section{Clinical presentation}

The demographic features of the nine patients comprising the study set are shown in Table 1 . Patients were generally young or middle aged (average age 44 years; range 32-68 years; male/ female $=4 / 5$ ) and most patients presented with shortness of breath, dyspnea on exertion, or a dry, nonproductive cough of long duration (average 1.8 years: range 6 months-4.2 years). Eight patients were current and long-standing cigarette smokers, with an average of 38 pack years (range 15-60 pack years; years of smoking 15-30). Pulmonary function studies were available on four patients and demonstrated mixed obstructive and restrictive lung disease, with predominantly obstructive lung disease with most patients having preserved lung volumes but reduced forced expiratory volume at $1 \mathrm{~s}$ and markedly reduced diffusing capacity. Chest radiographs and high resolution CT scans were performed on all nine patients of which only two were available for review. As shown in Table 1, the predominant patterns were bilateral micronodular or linear infiltrates. Ground glass infiltrates were seen in $44 \%$ of patients. Emphysema was also noted in four patients. No evidence of honeycomb fibrosis was described and traction bronchiectasis was not observed. All patients are alive with stable $(N=7)$ or slowly progressive $(N=2)$ disease at an average of 3.2 years follow-up (range $0.5-5.2$ years).

\section{Histologic features}

The nine biopsies all showed respiratory bronchiolitis. The predominant pattern was a centrilobular process with filling of terminal and respiratory bronchioles and centrilobular airspaces with finely pigmented macrophages (Figures 1 and 2). Mucous plugging was occasionally seen and was associated with mild atelectasis. A mild chronic inflammatory cell infiltrate surrounded the bronchioles and occasional lymphocytes and plasma cells were seen within alveolar septa. In addition to the respiratory bronchiolitis, the biopsies were remarkable for

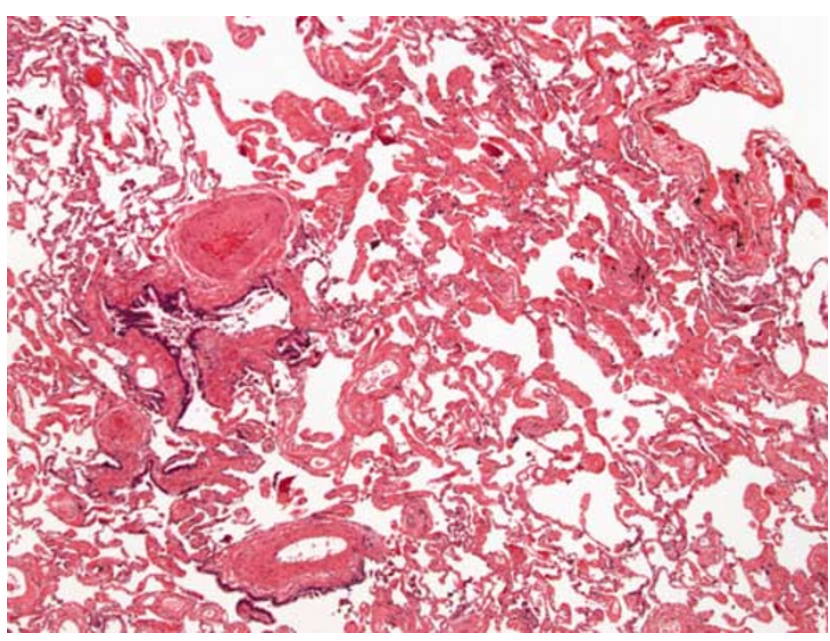

Figure 1 Respiratory bronchiolitis/interstitial lung disease with fibrosis. At scanning magnification, bronchioles and peribronchiolar airspaces contain smokers macrophages while centrilobular and subpleural architecture and structure is preserved although alveolar septa are irregularly thickened.

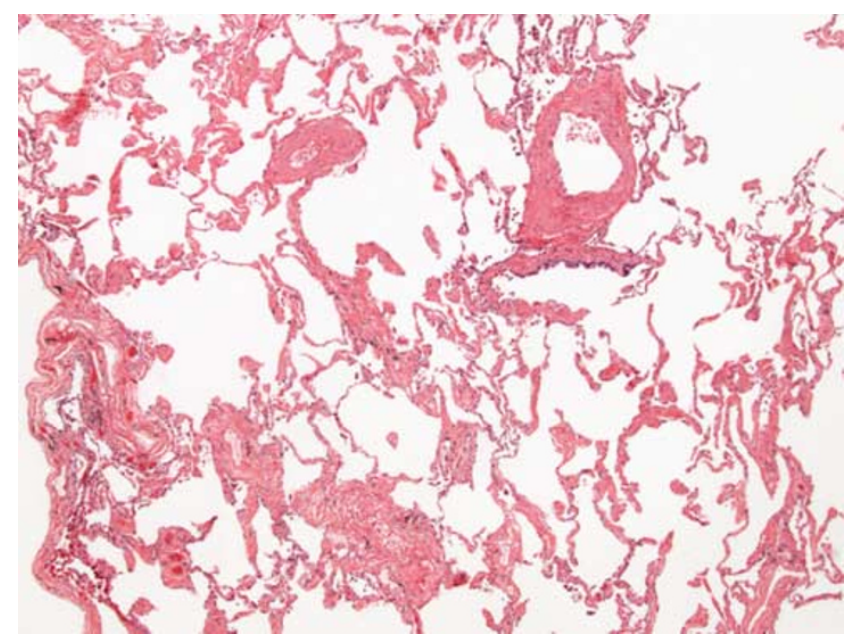

Figure 2 Respiratory bronchiolitis: interstitial lung disease with fibrosis. At $\times 4$, there is obvious irregular alveolar septal thickening in the setting of respiratory bronchiolitis and mild emphysematous change.

Table 1 Demographic information for respiratory bronchiolitis/interstitial lung disease cases with fibrosis

\begin{tabular}{lclll}
\hline Case & $\begin{array}{c}\text { Age/race/sex } \\
\text { (years) }\end{array}$ & Signs and symptoms & $\begin{array}{l}\text { Smoking history } \\
\text { (pk yrs) }\end{array}$ & CXR/HRCT (summary) \\
\hline$\# 1$ & $32 \mathrm{BM}$ & SOB, dyspnea & 60 & Bilateral micronodules/atelectasis \\
$\# 2$ & $50 \mathrm{WM}$ & SOB, cough & 44 & Bilateral micronodules/ground glass infiltrates \\
$\# 3$ & $38 \mathrm{WF}$ & Cough, wheeze, SOB, hemoptysis & 36 & Bilateral micronodules/emphysema \\
$\# 4$ & $44 \mathrm{WF}$ & Cough & 30 & Bilateral nodular densities/ground glass infiltrates \\
$\# 5$ & $43 \mathrm{WF}$ & SOB & 30 & Bilateral linear infiltrates/mosaic pattern/emphysema \\
$\# 6$ & $41 \mathrm{WF}$ & Malaise & 15 & Bilateral ground glass infiltrates \\
$\# 7$ & $47 \mathrm{BF}$ & SOB & 26 & Bilateral faint interstitial infiltrates \\
$\# 8$ & $46 \mathrm{WM}$ & DOE, wheeze, cough & 42 & Bilateral ground glass infiltrates/emphysema \\
$\# 9$ & $68 \mathrm{WM}$ & SOB &,+ Unknown & Emphysema; nonspecific interstitial markings \\
\hline
\end{tabular}

$\mathrm{B}=$ AfroAmerican; $\mathrm{W}=$ Caucasian; $\mathrm{SOB}=$ shortness of breath; $\mathrm{DOE}=$ dyspnea on exertion; $+=$ positive history; pk yrs $=$ pack years. 
expansion and thickening of the alveolar septa by pauci-cellular lamellae of dense eosinophilic collagen (Figure 3). This amyloid-like collagenous thickening of septa was widespread and more pronounced around the centrilobular zones but extended to the pleural surface in all cases in a patchy distribution (Figures 4 and 5). It was not accompanied by alveolar pneumocyte hyperplasia, bronchiolar metaplasia or airspace mucous inspissation, and Congo red stains were negative in four cases. This alveolar septal thickening was most dramatic in regions of enlarged airspaces that were crisscrossed by alveolar septa that were thickened and collagenized without a significant cellular infiltrate (Figure 6). The low power morphology

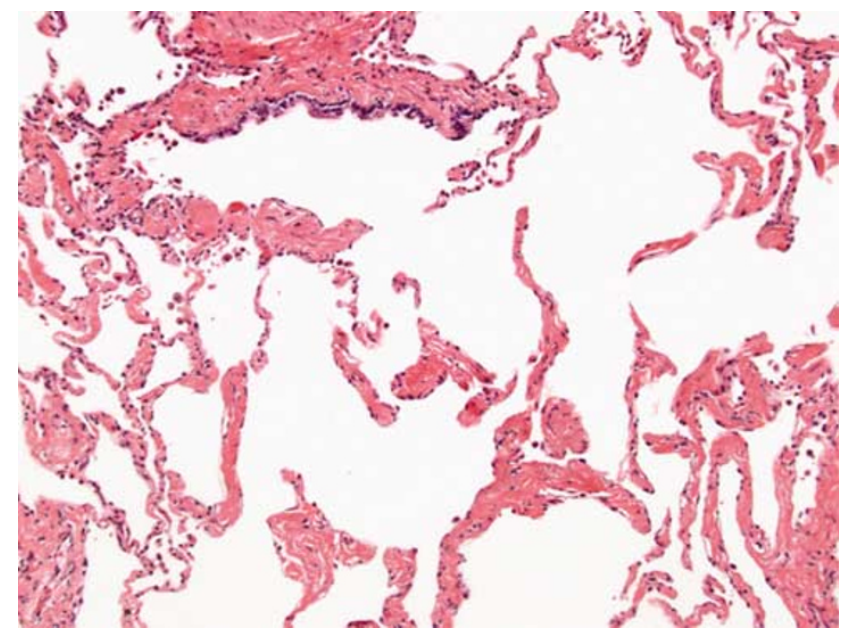

Figure 3 Respiratory bronchiolitis/interstitial lung disease with fibrosis. Respiratory bronchiolitis is accompanied by alveolar septa thickened by dense cords of ropey eosinophilic collagen with a scant mononuclear infiltrate.

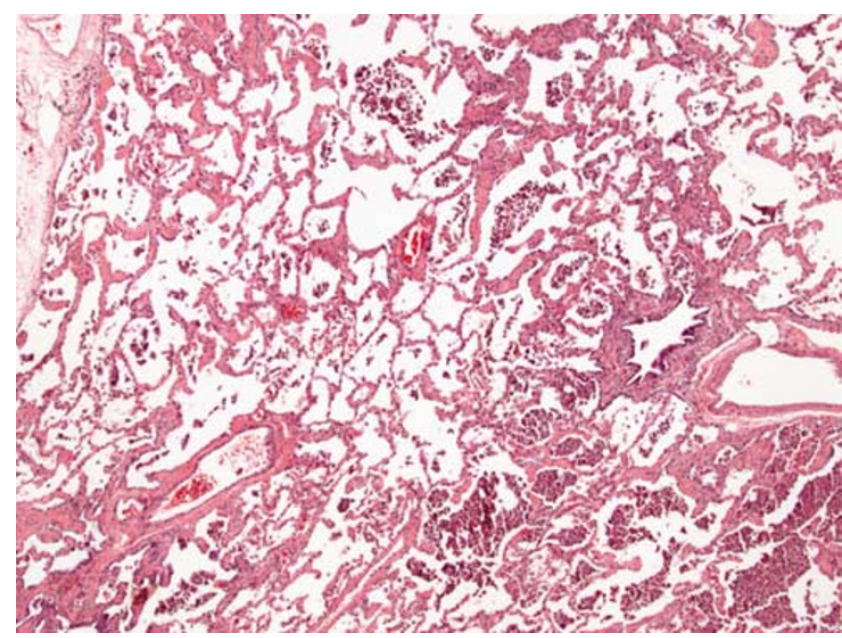

Figure 4 Respiratory bronchiolitis/interstitial lung disease with fibrosis. More extensive septal disease was characterized by diffuse septal fibrosis with retained parenchymal structure. Patchy airspace filling by smokers macrophages is seen. differed from honeycomb change because of the absence of bronchiolar metaplasia, thick fibrous septa and stasis of secretions and could best be characterized as emphysematous spaces traversed by these thickened and ropey fibrotic alveolar septa. Within these regions, occasional aggregates of pigmented macrophages were seen and, in fact, desquamative interstitial pneumonia-like change ( $3 \mathrm{~mm}$ in maximum dimension) was identified in three of the nine cases, with uniform filling of these airspaces by pigmented macrophages with diffuse alveolar septal collagenization (Figure 7).

Lymphoid aggregates were seen in three cases and a pulmonary apical cap was noted as an incidental finding in one case.

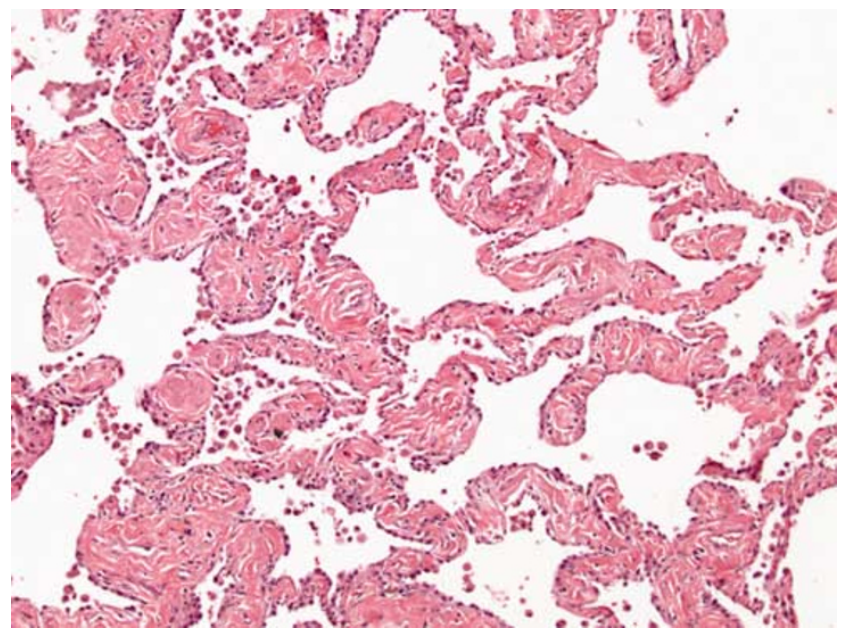

Figure 5 Respiratory bronchiolitis/interstitial lung disease with fibrosis. The thickened septa were composed of amyloid-like lamellae of dense ropey eosinophilic collagen, with mild prominence of alveolar lining cells but no bronchiolar metaplasia or honeycomb change.

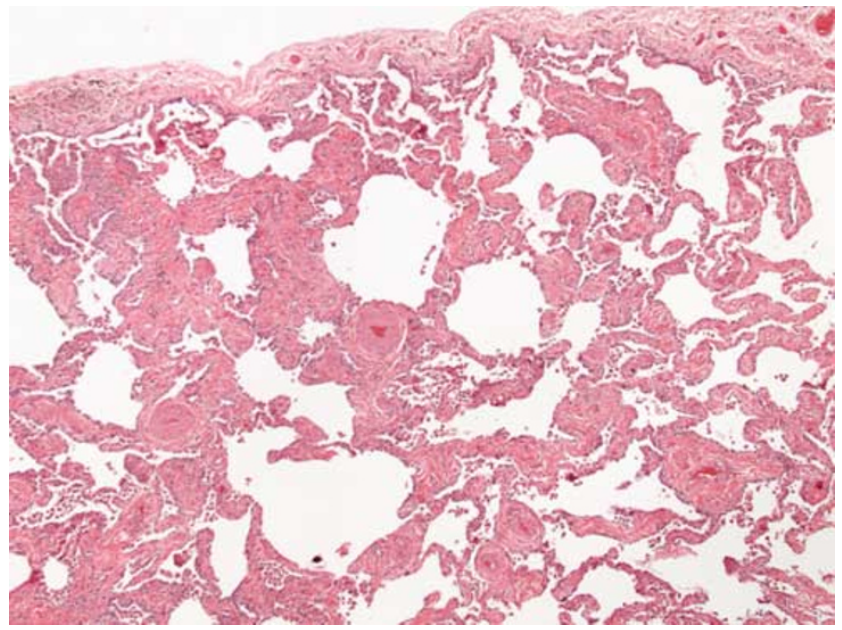

Figure 6 Respiratory bronchiolitis/interstitial lung disease with fibrosis. Focal areas of diffuse alveolar septal fibrosis extending to the pleural surface were seen in all cases, occasionally accompanied by emphysematous change, and resembled fibrotic nonspecific interstitial pneumonia. 


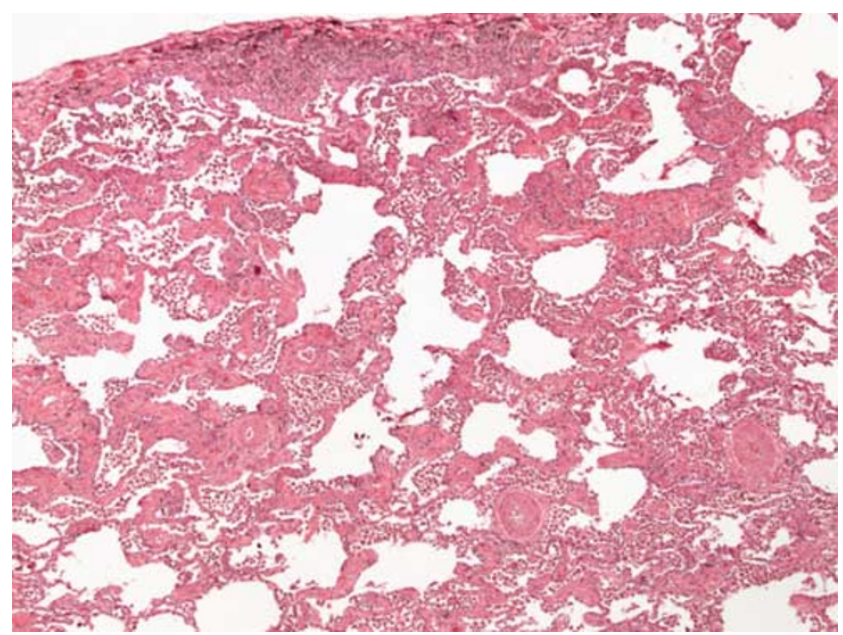

Figure 7 Respiratory bronchiolitis with fibrosis. In $30 \%$ of the cases, desquamative interstitial pneumonia-like areas of uniform airspace filling by smokers' macrophages were seen in association with septal fibrosis.

Specific negative findings included the absence of organizing pneumonia or interstitial fibroblastic foci, honeycomb changes (as noted above), granulomas or mucous plugging of airspaces.

As part of this study, the grossly sectioned 'normal' lung of thirty consecutive lobectomies performed on known cigarette smokers with nonsmall-cell lung carcinoma (18 adenocarcinoma, 12 squamous carcinoma) without evidence of intersitial lung disease on routine CT scans, were examined. In all, 17 (56\%) showed respiratory bronchiolitis without evidence of alveolar septal fibrosis. Six $(20 \%)$ had respiratory bronchiolitis with mild occasional irregular skeins of septal fibrosis localized to the centrilobular zones with three $(10 \%)$ having cords of thick acellular septal collagen forming a focal lattice within the mid zone of at least one lobule. In four cases (14\%), the respiratory bronchiolitis had the alveolar septal fibrosis extend to the subpleural regions or interlobular septa, and had a morphologic appearance that focally resembled the cases of respiratory bronchiolitis-associated interstitial lung disease with fibrosis described herein.

As additional control groups for these morphologic features, we reviewed 16 age-matched pneumonectomies of nonsmokers with primary pulmonary hypertension who received lung transplants and lobectomies from seven nonsmoking patients (six female, one male) with primary lung adenocarcinomas. In none of these cases did we identify the characteristic ropey eosinophilic collagenous thickening of the alveolar ducts and alveolar septa described in the nine study cases or the first control group of lobectomies in smokers. Similarly, pigmented alveolar macrophages of the 'smokers' type were not seen.

\section{Discussion}

The nine cases of respiratory bronchiolitis-associated interstitial lung disease with fibrosis that comprise this series highlight one component of its histopathologic spectrum that may cause diagnostic confusion-specifically the presence of significant peribronchiolar and subpleural collagenous fibrosis of the alveolar septa such that the contributing pathologists considered a diagnosis of fibrotic phase nonspecific interstitial pneumonia. This was a particularly difficult distinction because to some extent the clinical presentation, functional data and radiologic features of the two entities overlap, particularly in cases of nonspecific interstitial pneumonia occurring in cigarette smokers.

Since the early descriptions of respiratory bronchiolitis in autopsy series, many studies have emphasized the presence of finely pigmented macrophages within the lumens of small airways and in the centrilobular airspaces, as a consistent marker of exposure to tobacco smoke. ${ }^{1-7,9,11-14,16-18}$ Histologic descriptions have indicated peribronchiolar fibrosis and chronic inflammation but the best description by Fraig et $a l^{16}$ found that nearly $50 \%$ of respiratory bronchiolitis cases have focal mild alveolar septal fibrosis and yet this observation did not consistently translate into evidence of radiographic changes or functional deficits. This is similar to the cases presented herein which are more remarkable for the widespread dense amyloid-like collagenous thickening of the entire lobule, particularly centrilobular and subpleural alveolar septa. The consequence of this interstitial fibrosis was a pattern of eosinophilic pauci-cellular collagenization of septa that was unassociated with the parenchymal remodeling of honeycomb change, fibroblastic foci or significant chronic inflammation. The nine cases appear to be cases that reflect the severe end of the spectrum of changes noted in cigarette smokers; these distinctive histopathologic changes are usually mild and incidental as shown in the study by Fraig et $a l^{16}$ and as found in the control group of lobectomies in smokers in this study. At the severe end of the spectrum these changes may be associated with symptoms, signs and radiologic findings of interstitial lung disease. Based on these observations we think these cases should be segregated from the category of fibrotic nonspecific interstitial pneumonia and considered one of the manifestations of smoking-related interstitial lung disease/ respiratory bronchiolitis-associated interstitial lung disease with fibrosis. The presence of a fibrotic process in the lung of these patients, in the absence of features of usual interstitial pneumonia, led to a consideration of the fibrotic form of nonspecific interstitial pneumonia. ${ }^{19-26}$ This was especially true in the areas of subpleural emphysema where enlarged emphysematous airspaces were distinctly defined by thickened fibrotic alveolar septa and occasionally interlobular septa forming linear 
cicatrixes oriented perpendicular to the axis of the visceral pleura. Superficially, this pattern resembled microscopic honeycomb change because of the coexistent loss of alveolar septa due to emphysema. It did, however, lack bronchiolar metaplasia, true fibrous parenchymal remodeling and broad bands of pulmonary parenchymal scarring, and mucous inspissation.

The cases of respiratory bronchiolitis-associated interstitial lung disease with fibrosis in this study, and the associated control groups, demonstrate that cigarette smoking is associated with airway inflammation and with a patchy yet extensive, dense paucicellular collagenous scarring of alveolar septa. ${ }^{8,9,11,17}$ It contrasts with historical perceptions that smoking cannot be associated with extensive fibrosis of alveolar septa, resulting instead in dissolution of septa and emphysematous change. This observation has been noted previously in smokers in pathologic and radiologic studies ${ }^{3,6,14,16}$ and these nine cases show that respiratory bronchiolitisassociated interstitial lung disease with fibrosis can be of such an extent as to resemble the fibrotic phase of nonspecific interstitial pneumonia, especially when the interstitial process is patchy as described by Kazenstein et al. ${ }^{24}$ This distinction can be a difficult one because the clinical and radiographic features of these cases, 'classical' respiratory bronchiolitis-associated interstitial lung disease and fibrotic phase nonspecific interstitial pneumonia in a smoker show substantial overlap with symptomatic dyspnea and cough, mixed functional deficits, and radiographic abnormalities of centrilobular micronodules and ground glass infiltrates being characteristic. ${ }^{13,24,26}$ In a minority of cases, nonspecific interstitial pneumonia patients may have honeycomb change and tend to have more extensive ground glass changes. Morphologic features that are particularly useful in nonspecific interstitial pneumonia include a more diffuse distribution of interstitial fibrosis and a more pronounced mononuclear inflammatory infiltrate that is absent in respiratory bronchiolitis-associated interstitial lung disease with fibrosis. In fibrotic nonspecific interstitial pneumonia, the fibrosis lacks the dense lamellar type collagenization of alveolar septa, and the septal changes are often accompanied by prominent type II alveolar pneumocytes, patches of fibroblastic foci; and in a minority honeycomb change. Microscopic honeycomb change is associated with remodeled, not emphysematous lung, where septa are thick, airspaces reduced in size, bronchiolar metaplasia is prominent, and mucinous plugging often with acute inflammation is seen. The relatively poor prognosis of fibrotic nonspecific interstitial pneumonia compared to our cases warrants recognition of this differential diagnostic entity.

Although the emphasis of this series is on the mimicry of respiratory bronchiolitis-associated interstitial lung disease with nonspecific interstitial pneumonia, a major differential diagnostic con- sideration includes desquamative interstitial pneumonia. ${ }^{3,6-10,12,13,15,27}$ Desquamative interstitial pneumonia is defined as having widespread diffuse alveolar septal fibrosis with uniform airspace filling by alveolar macrophages and most examples of desquamative interstitial pneumonia are felt to represent one extreme of tobacco induced lung disease with respiratory bronchiolitis at the other pole. As noted by Ryu et $a l,{ }^{13}$ desquamative interstitial pneumonia usually displays more extensive ground glass changes, more severe functional impairment, has more striking symptomatology including clubbing and has an acknowledged, albeit low mortality. These cases of respiratory bronchiolitis-associated interstitial lung disease with fibrosis probably fall somewhere within the above spectrum where the alveolar septal fibrosis is dramatic and more extensive than usual cases of respiratory bronchiolitis-associated interstitial lung disease, but lack the diffuse uniform airspace filling by macrophages. To some extent they represent alveolar macrophage 'poor' cases of desquamative interstitial pneumonia, more resembling the fibrotic form of nonspecific interstitial pneumonia.

Peribronchiolar fibrosis is also seen with Langerhans cell histiocytosis in its late 'burnt' out phase, however, the extent and shape of the stellate scars, the frequent presence of sheets of Langerhans cells in younger lesions, and the presence of such scars in noncentrilobular locations can be helpful discriminators.

In summary, this series of nine cases of respiratory bronchiolitis-associated interstitial lung disease with fibrosis highlights an important observation for the surgical pathologist-an interstitial fibrosis can develop as a result of tobacco use, and this process needs to be separated from fibrotic forms of nonspecific interstitial pneumonia especially those occurring in cigarette smokers.

\section{Acknowledgements}

We wish to thank Diana Winters for secretarial assistance and Linda Shab for photographic aid.

\section{References}

1 Adesina AM, Vallyathan V, McQuillen EN. Bronchiolar inflammation and fibrosis associated with smoking: a morphologic cross-section population analysis. Am Rev Respir Dis 1991;143:144-149.

2 Agius RM, Rutman A, Knight RK, et al. Human pulmonary alveolar macrophages with smokers' inclusions: their relation to the cessation of cigarette smoking. Br J Ex Pathol 1986;67:407-413.

3 Myers JL, Veal CF, Shin MS, et al. Respiratory bronchiolitis causing interstitial lung disease. Am Rev Respir Dis 1987;135:880-884.

4 Niewoehner DE, Kleinerman J, Rice DB. Pathologic changes in the peripheral airways of young cigarette smokers. N Engl J Med 1974;291:755-758. 
5 Wright JL, Lawson LM, Pare PD, et al. Morphology of peripheral airways in current smokers and ex-smokers. Am Rev Respir Dis 1983;127:474-477.

6 Yousem SA, Colby TV, Gaensler EA. Respiratory bronchiolitis associated interstitial lung disease and its relationship to desquamative interstitial pneumonia. Mayo Clin Proc 1989;64:1373-1380.

7 Davies G, Wells AU, Du Bois RM. Respiratory bronchiolitis associated with interstitial lung disease and desquamative interstitial pneumonia. Clin Chest Med 2004;25:717-726.

8 Hansell DM, Nicholson AG. Smoking-related diffuse parenchymal lung disease: HRCT-pathologic correlation. Sem Resp Crit Care M 2003;24:377-391.

9 Heyneman LE, Ward S, Lynch DA, et al. Respiratory bronchiolitis, respiratory bronchiolitis-associated interstitial lung disease, and desquamative interstitial pneumonia: different entities or part of the spectrum of the same disease process. Am J Roetgenol 1999; 173:1617-1622.

10 Moon J, du Bois RM, Colby TV, et al. Clinical significance of respiratory bronchiolitis on open lung biopsy and its relationship to smoking related interstitial lung disease. Thorax 1999;54:1009-1014.

11 Park JS, Brown KK, Tuder RM, et al. Respiratory bronchiolitis-associated interstitial lung disease: radiologic features with clinical and pathologic correlations. J Comput Assist Tomogr 2002;26:13-20.

12 Remy-Jardin M, Remy J, Gosselin B, et al. Lung parenchymal changes secondary to cigarette smoking: pathologic-CT correlations. Radiology 1993;186: 643-651.

13 Ryu JH, Myers JL, Capizzi SA, et al. Desquamative interstitial pneumonia and respiratory bronchiolitis associated interstitial lung disease. Chest 2005; 127:178-184.

14 Wells AU, Nicholson AG, Hansell DM, et al. Respiratory bronchiolitis-associated interstitial lung disease. Sem Resp Crit Care M 2003;24:585-594.

15 Craig PJ, Wells AU, Doffman S, et al. Desquamative interstitial pneumonia, respiratory bronchiolitis and their relationship to smoking. Histopathology 2004;45: 275-282.

16 Fraig M, Shreesha U, Savici D, et al. Respiratory bronchiolitis: a clinicopathologic study in current smokers, ex-smokers, and never-smokers. Am J Surg Pathol 2002;26:647-653.

17 Cosio MG, Hale KA, Niewoehner DE. Morphologic and morphometric effects of prolonged cigarette smoking on the small airways. Am Rev Respir Dis 1980;122: $265-271$.

18 Cottin V, Streichenberger N, Gamondes J-P. Respiratory bronchiolitis in smokers with spontaneous pneumothorax. Eur Respir J 1998;12:702-704.

19 Cottin V, Donsbeck A-V, Revel D, et al. Nonspecific interstitial pneumonia: individualizing of a clinicopathologic entity in a series of 12 patients. Am J Respir Crit Care Med 1998;158:1286-1293.

20 Daniil ZD, Gilchrist FC, Nicholson AG, et al. A histologic pattern of nonspecific interstitial pneumonia is associated with a better prognosis than usual interstitial pneumonia in patients with cryptogenic fibrosing alveolitis. Am J Respir Crit Care Med 1999; 160:899-905.

21 Flaherty K, Toews F, Travis W, et al. Clinical significance of histological classification of idiopathic interstitial pneumonia. Eur Respir J 2002;19:275-283.

22 Flaherty KR, Martinez FJ, Travis WD, et al. Nonspecific interstitial pneumonia (NSIP). Sem Resp Crit Care Med 2001;22:423-433.

23 Hartman TE, Swensen SJ, Hansell DM, et al. Nonspecific interstitial pneumonia: variable appearance at high-resolution chest CT. Radiology 2000;217: 701-705.

24 Katzenstein A-L, Fiorelli RF. Nonspecific interstitial pneumonia/fibrosis: histologic features and clinical significance. Am J Surg Pathol 1994;18:136-147.

25 Katzenstein A-L, Myers JL. Nonspecific interstitial pneumonia and the other idiopathic interstitial pneumonias: Classification and Diagnostic Criteria. Am J Surg Pathol 200l;24:1-3.

26 Travis WD, Matsui K, Moss J, et al. Idiopathic nonspecific interstitial pneumonia: prognostic significance of cellular and fibrosing patterns: survival comparisons with usual interstitial pneumonia and desquamative interstitial pneumonia. Am J Surg Pathol 2000;24:19.

27 Bedrossian CWM, Kuhn CI, Luna MA. Desquamative interstitial pneumonia-like reaction accompanying pulmonary lesions. Chest 1977;72:166-169. 